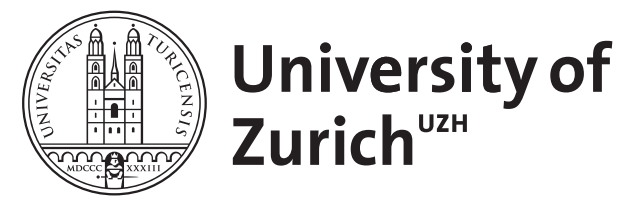

\title{
Fate of mountain glaciers in the anthropocene
}

Bengtsson, L ; Breashears, D ; Crutzen, P J ; Fuzzi, S ; Haeberli, W ; Immerzeel, W W ; Kaser, G ; Kennel, C ; Kulkarni, A ; Pachauri, R ; Painter, T H ; Rabassa, J ; Ramanathan, V ; Robock, A ; Rubbia, C ; Russell, L ; Sánchez Sorondo, M ; Schellnhuber, H J ; Sorooshian, S ; Stocker, T F ; Thompson, L G ; Toon, O B ; Zaelke, D ; Mittelstrass, J

Posted at the Zurich Open Repository and Archive, University of Zurich ZORA URL: https://doi.org/10.5167/uzh-50863

Conference or Workshop Item

Published Version

Originally published at:

Bengtsson, L; Breashears, D; Crutzen, P J; Fuzzi, S; Haeberli, W; Immerzeel, W W; Kaser, G; Kennel, C; Kulkarni, A; Pachauri, R; Painter, T H; Rabassa, J; Ramanathan, V; Robock, A; Rubbia, C; Russell, L; Sánchez Sorondo, M; Schellnhuber, H J; Sorooshian, S; Stocker, T F; Thompson, L G; Toon, O B; Zaelke, D; Mittelstrass, J (2011). Fate of mountain glaciers in the anthropocene. In: Workshop on Fate of Mountain Glaciers in the Anthropocene, Vatican, 2 April 2011 - 4 April 2011, Pontificial Academy of Sciences. 


\section{Das Schicksal der Gebirgsgletscher im Anthropozän}

Ein Bericht der Arbeitsgruppe im Auftrag der Päpstlichen Akademie der Wissenschaften
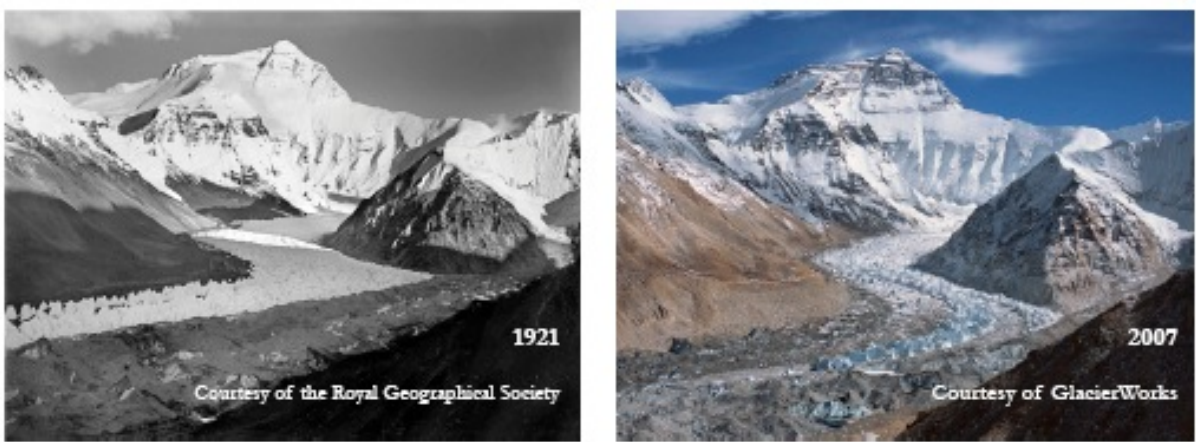

Rongbuk-Hauptgletscher (vgl. Innenseite)

11. Mai 2011

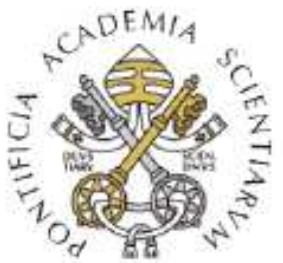

Die Arbeitsgruppe besteht aus Glaziologen, Klimatologen, Meteorologen, Hydrologen, Physikern, Chemikern, Bergsteigern und Juristen. Sie wurde von der Päpstlichen Akademie der Wissenschaften am Vatikan eingerichtet, um den beobachteten Rückgang der Gebirgsgletscher, seine Gründe und Folgen eingehend zu erörtern. Dieser Bericht ist das Ergebnis eines Arbeitskreises im April 2011 im Vatikan. 
Titelbild:

Rongbuk-Hauptgletscher

Lage: Mount Everest, 8848 m, Autonomes Gebiet Tibet

Gebirgskette: Mahalangur Himal, Ost-Himalaya

Geographische Koordinaten: $27^{\circ} 59^{\prime} 15^{\prime \prime} \mathrm{N}, 86^{\circ} 55^{\prime} 29^{\prime \prime}$ Ost

Höhenlage des Gletschers: $5060 \mathrm{~m}-6462 \mathrm{~m}$

Durchschnittlicher Dickenverlust des Gletschers: $101 \mathrm{~m}$ im Zeitraum von1921 bis 2008 

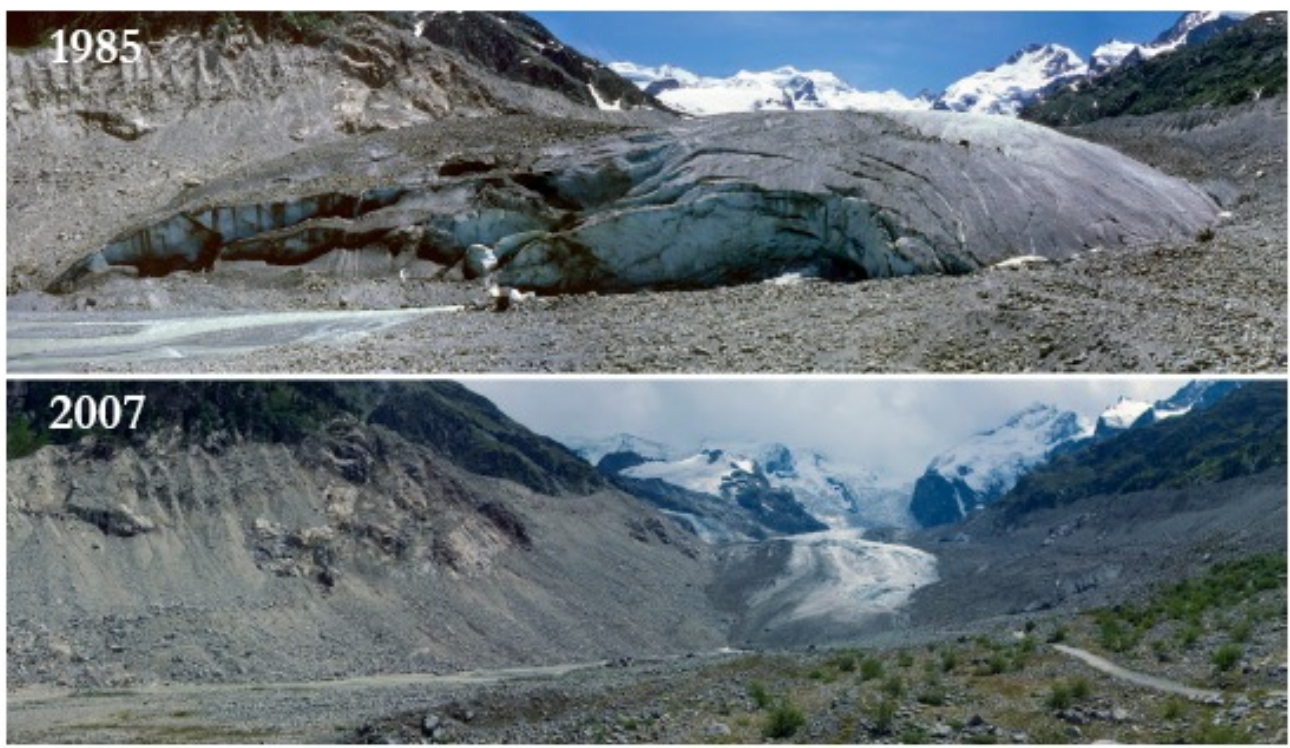

Morteratsch Gletscher (Alpen). Mit freundlicher Genehmigung von J. Alean, SwissEduc

\section{Erklärung der Arbeitsgruppe}

Wir rufen alle Menschen und Nationen auf, die schwerwiegenden und möglicherweise unumkehrbaren Auswirkungen der globalen Erwärmung zu erkennen, die durch die anthropogene Emission von Treibhausgasen und anderen Umweltschadstoffen ebenso wie durch Veränderungen von Wäldern, Feuchtgebieten, Grünland und anderen Landnutzungsarten verursacht werden. Wir appellieren an alle Nationen, unverzüglich wirksame und ausreichende Maßnahmen zu entwickeln und umzusetzen, um die Ursachen und Auswirkungen des Klimawandels auf Gemeinschaften und Ökosysteme unter Einbeziehung der Gebirgsgletscher und ihrer Einzugsgebiete zu verringern, da wir uns dessen bewusst sind, dass es nur eine gemeinsame Heimat für uns alle gibt. Wenn wir jetzt im Sinn unserer gemeinschaftlichen, wiewohl unterschiedlich verteilten Verantwortung handeln, bejahen wir unsere Verpflichtung gegenüber unseren Mitmenschen und übernehmen die Verantwortung für einen Planeten, der mit dem Geschenk des Lebens gesegnet ist.

Wir sind verpflichtet, die Versorgung aller Bewohner dieses Planeten mit täglichem Brot, frischer Atemluft und sauberem Trinkwasser sicherzustellen. Denn wenn wir Gerechtigkeit und Frieden wollen, müssen wir unseren Lebensraum schützen. Die Gläubigen unter uns bitten Gott, uns diesen Wunsch zu erfüllen. 



\section{Zusammenfassung}

Zurückgehende Gletscher erfordern dringende Reaktionen

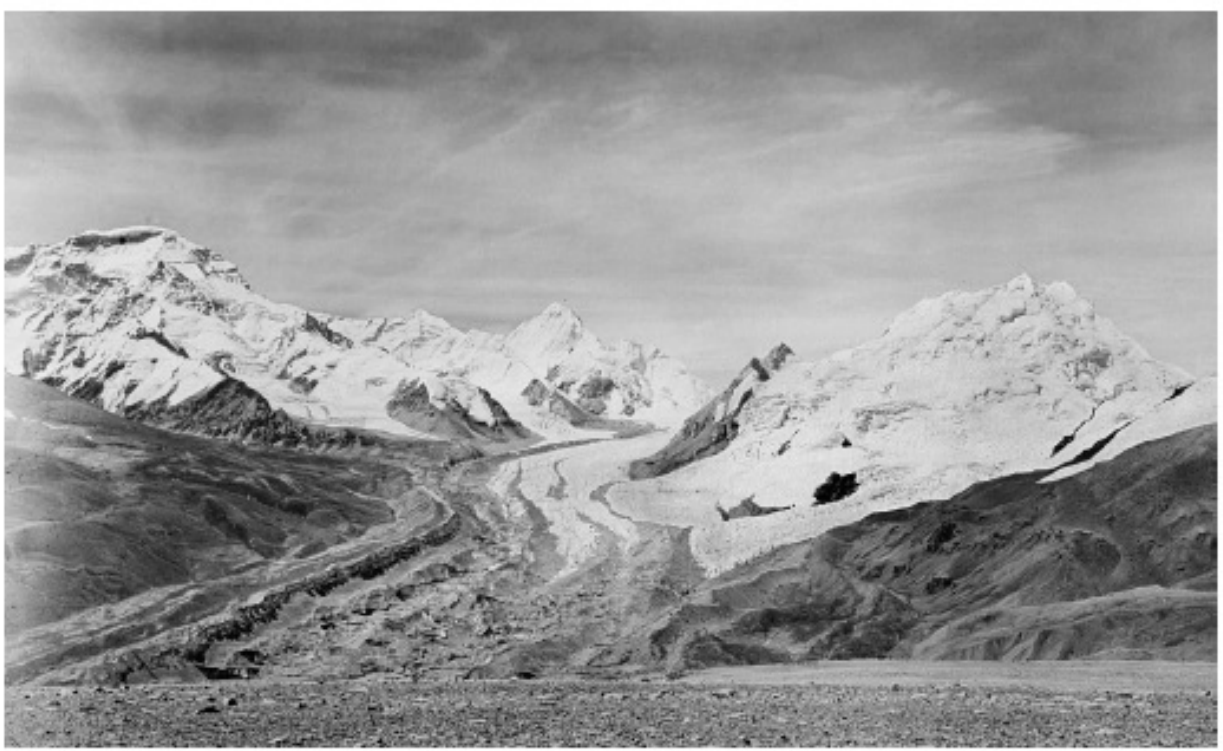

Kyetrak Gletscher 1921. Lage: Cho Oyu, 8201 m, Autonomes Gebiet Tibet; OstHimalaya. Höhenlage des Gletschers: 4907 m-5883 m. Mit freundlicher Genehmigung der Royal Geographical Society.

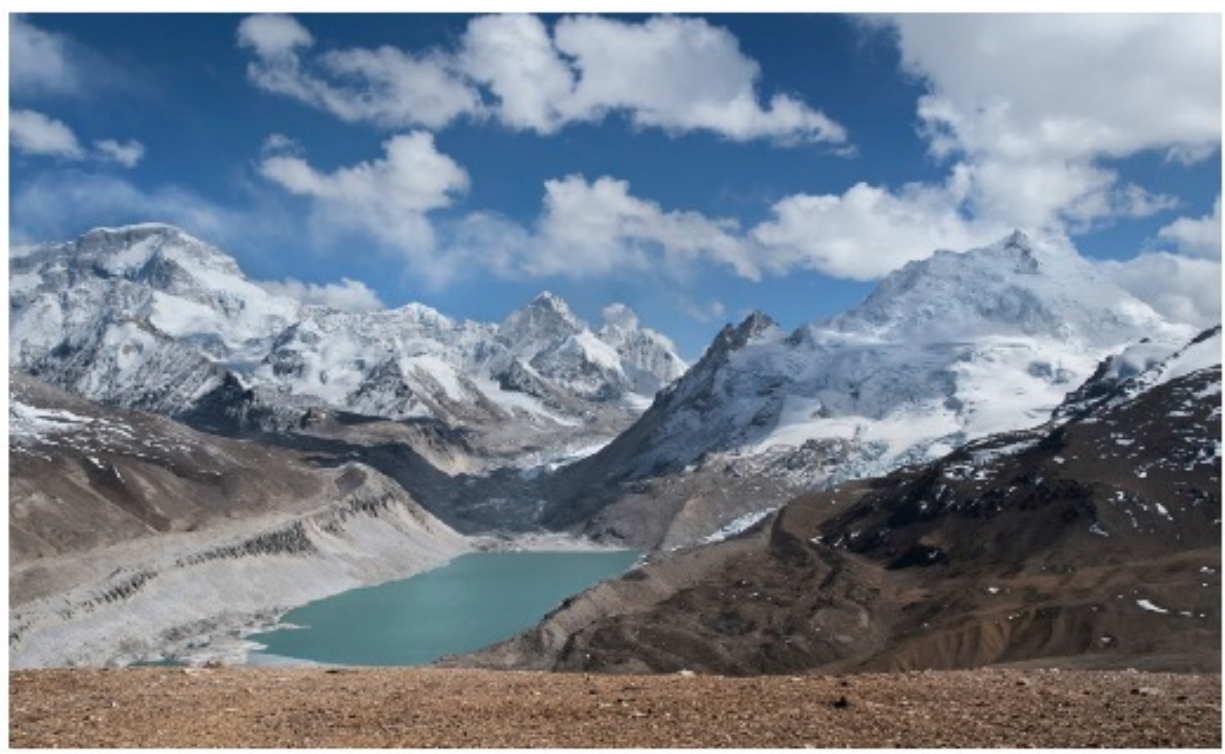

Kyetrak Gletscher 2009; Fotographie aus dem Jahr 2009: mit freundlicher Genehmigung von Glacier Works. 
Anthropozän: Die offensive Ausbeutung fossiler Brennstoffe und anderer natürlicher Ressourcen hat die Luft, die wir atmen, das Wasser, das wir trinken und das Land, das wir bewohnen, geschädigt. So wurden zum Beispiel etwa 1000 Milliarden Tonnen Kohlendioxid und andere klimatisch bedeutsame Treibhausgase in die Atmosphäre abgegeben mit dem Ergebnis, dass die Kohlendioxid-Konzentration in der Luft jetzt die höchsten Werte der letzten 800000 Jahre aufweist. Die klimatischen und ökologischen Auswirkungen dieses menschlichen Eingriffs in das Erdsystem werden voraussichtlich viele Jahrtausende andauern und rechtfertigen damit einen neuen Namen, nämlich „Das Anthropozän“ für die neue, menschengemachte geologische Epoche, in der wir leben.

Rückgang der Gletscher: Weltweit gehen die Gletscherflächen zurück, und die höchsten Verlustraten werden in niedrigen Meereshöhen verzeichnet. Mit dem weitverbreiteten Schwund von Gletschern, Eis und Schnee in den Gebirgen der tropischen, gemäßigten und polaren Regionen verfügen wir über einen der deutlichsten Beweise für einen Wandel im Klimasystem, der sich mit hoher Geschwindigkeit global vollzieht. Langfristige Datenreihen zeigen, dass sich die Massenverlustrate seit dem Jahrhundertwechsel mehr als verdoppelt hat. Schmelzwasser von Gletschern und vom Schnee im Gebirge haben wesentlich zu dem im letzten Jahrhundert beobachteten Meeresspiegelanstieg beigetragen. Ein Rückgang der Gletscher in den Europäischen Alpen wird seit dem Ende der „Kleinen Eiszeit“ in der ersten Hälfte des 19. Jahrhunderts beobachtet. Die Geschwindigkeit des Rückgangs aber war seit den 1980-er Jahren erheblich höher als vorher. Die Alpengletscher haben inzwischen mehr als die Hälfte ihrer Masse verloren. Tausende kleine Gletscher in der Hindukush-Himalaya-Tibet Region lösen sich immer mehr auf und bilden damit eine Bedrohung für örtliche Gemeinden und die noch zahlreicheren weiter entfernt lebenden Menschen, die von den Wasserressourcen der Berge abhängen. Verlässliche Szenarienberechnungen zeigen klar, dass viele Gebirgsketten in der ganzen Welt den größeren Teil ihrer Gletscher im Lauf der nächsten Jahrzehnte verlieren könnten.

Die in jüngster Vergangenheit beobachteten Veränderungen des Gletscherverhaltens sind bedingt durch eine komplexe Mischung von Ursachen, welche Treibhausgas-Antriebe ebenso beinhalten wie großräumige Emissionen von dunklen Rußpartikeln und Staub in „braunen Wolken“, mit allen damit verbundenen Veränderungen des regionalen atmosphärischen Energie- und Feuchtegehalts. Das alles resultiert in einer deutlichen Erwärmung in größeren Höhen, nicht zuletzt im Himalaya.

Ein Blick auf frühere Veränderungen: Als Erwiderung auf das Argument, dass „die heutigen Veränderungen des Klimas und der Eisbedeckung völlig natürliche Vorgänge sind, weil die Erde wechselnde Kaltzeiten (Eiszeiten oder Glaziale) und Warmzeiten (Interglaziale) bereits in der Vergangenheit erlebt hat", halten wir fest:

Die primär auslösenden Faktoren für Eiszeiten und Interglaziale liegen bekanntlich in Änderungen der astronomischen Parameter, die mit der Bewegung der Erde im Sonnensystem verbunden sind, und in natürlichen Rückkopplungsprozessen im Klimasystem. Die Zeitskalen dieser auslösenden Faktoren liegen im Bereich von 10000 Jahren oder länger. Im Gegensatz dazu finden die beobachteten menschengemachten Änderungen von Kohlendioxid, anderen Treibhausgasen und Rußkonzentrationen auf einer Zeitskala von 10 bis 100 Jahren statt-also mindestens hundertmal so schnell. Besonders beunruhigend ist die Tatsache, dass diese Freisetzung von Stoffen, welche die globale Erwärmung steuern, während eines Interglazials stattfindet, in dem sich die Erde bereits auf einem natürlichen Temperaturmaximum befand. 
Wir empfehlen drei Maßnahmen: Vom Menschen verursachte Veränderungen in der Zusammensetzung der Luft und der Luftqualität haben weltweit mehr als 2 Millionen vorzeitige Todesfälle pro Jahr zur Folge und bedrohen die Sicherheit von Wasser und Nahrung - besonders bei den „drei Milliarden Menschen auf der niedrigsten sozialen Stufe“, die zu arm sind um den Schutz fossiler Brennstoffe und der Industrialisierung nutzen zu können. Da eine nachhaltige Zukunft, die auf der weiteren Ausbeutung von Kohle, Erdöl und Erdgas in der „business-as-usual“ Methode basiert, sowohl wegen der Erschöpfung der Ressourcen als auch wegen der Umweltschäden (verursacht z.B. durch den gefährlichen Meeresspiegelanstieg) nicht möglich ist, fordern wir unsere Gesellschaften dringend auf,

I. Die weltweiten Kohlendioxid-Emissionen unter Einsatz aller denkbaren Methoden unverzüglich zu reduzieren, um die ehrgeizigen internationalen ErwärmungsSollwerte zu erfüllen und die Langzeitstabilität des Klimasystems zu sichern. Alle Nationen müssen sich auf einen schnellen Übergang zu erneuerbaren Energiequellen und auf andere Strategien zur Reduktion der $\mathrm{CO}_{2}$-Emissionen konzentrieren. Die Staaten sollten auch den Abbau der Kohlenstoffsenken verhindern, indem sie die Abholzung von Wäldern beenden, und Kohlenstoffsenken z.B. durch die Wiederaufforstung von degradierten Landflächen verstärken. Sie müssen auch Technologien entwickeln und anwenden, um überschüssiges Kohlendioxid aus der Atmosphäre wieder zu entfernen. Diese Maßnahmen müssen innerhalb einiger weniger Dekaden erfolgen.

II. Die Konzentrationen von zur Erwärmung beitragenden Umweltschadstoffen (dunkler Ruß, Methan, troposphärisches Ozon, Fluorkohlenwasserstoffe) um bis zu 50\% zu senken, um den Klimawandel in diesem Jahrhundert zu verlangsamen und damit Millionen vorzeitiger Todesfälle zu verhindern, die von Atemwegserkrankungen und Millionen Tonnen Ernteausfällen pro Jahr verursacht werden.

III. Sich auf langsam verlaufende ebenso wie auf sehr schnell erfolgende Klimaveränderungen einzustellen, die von der Gesellschaft nicht verhindert werden

können. Insbesondere fordern wir eine Initiative für eine weltweite Erweiterung der Fachkompetenzen, um die natürlichen und die sozialen Auswirkungen des Klimawandels in Gebirgssystemen und den damit verbundenen Wasser-Einzugsgebieten abzuschätzen.

Die Kosten dieser drei Empfehlungen verblassen im Vergleich zu dem Preis, den die Welt bezahlen muss, wenn wir es jetzt unterlassen zu handeln. 


\section{Wissenschaftliche Erkenntnisse und Empfehlungen}

Der anhaltende Klimawandel stellt eine tödliche Gefahr für Gebirgsgletscher dar
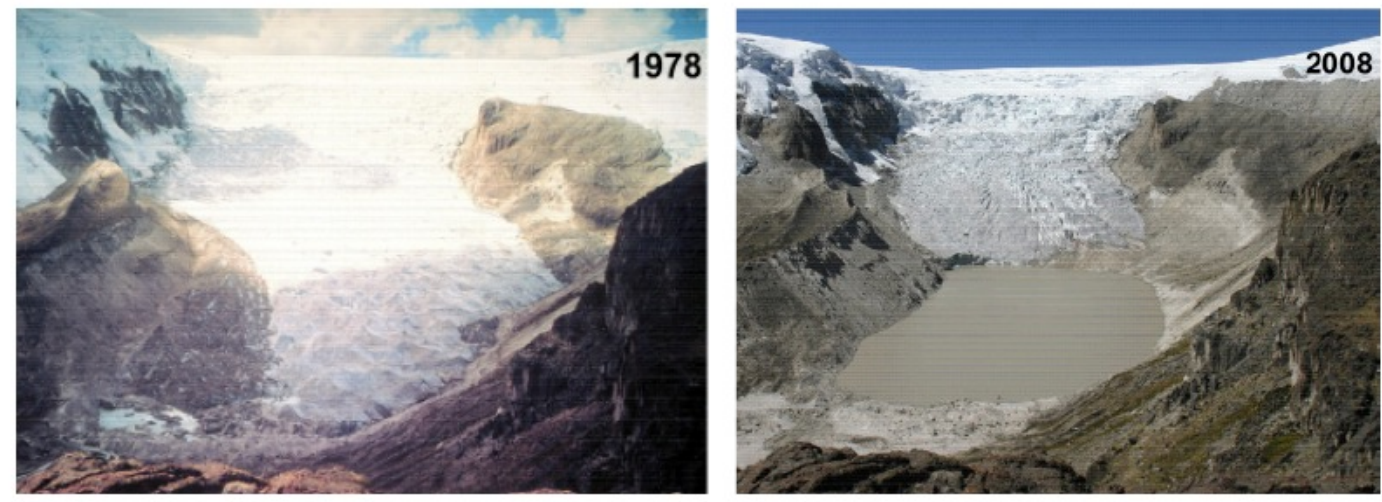

Qori Kalis Gletscherstrom (größter Gletscherstrom aus dem Quelcaya Inlandeis der südlichen peruanischen Anden). Mit freundlicher Erlaubnis von Lonnie Thompson, Ohio State Universität

\section{Das Anthropozän: Eine neue geologische Epoche}

Während der letzten zwei Jahrhunderte fand eine beispiellose Zunahme der Erdbevölkerung ebenso wie der Ausbeutung der globalen Ressourcen statt. Dieser Raubbau hat zunehmend negative Einflüsse auf viele Komponenten des Erdsystems - auf die Luft, die wir atmen, das Wasser, das wir trinken und das Land, das wir bewohnen. Die Menschheit verändert das Klimasystem durch die Emission von Treibhausgasen und von Wärme absorbierenden Umweltverschmutzungsstoffen. Die derzeitige atmosphärische Konzentration von Kohlendioxid als dem Haupt-Treibhausgas übersteigt alle anderen Maximalwerte während der letzten 800000 Jahre. Ausgedehnte Umwandlungen der Landoberfläche, die Verluste an Waldflächen, Grünland, Feuchtgebieten und anderen Ökosystemen beinhalten, verursachen ebenfalls einen Klimawandel. Nobelpreisträger Paul Crutzen hat dieser neuen, von uns selbst geschaffenen geologischen Epoche den Namen „Anthropozän“ verliehen, angesichts der Tatsache, dass diese Komponenten des Erdsystems durch menschliches Handeln grundlegend verändert werden.

Unter dem Patronat der Päpstlichen Akademie der Wissenschaften traf sich vom 2. bis 4. April 2011 eine wissenschaftliche Expertengruppe in der ,Casina Pius IV' im Vatikan, um das Schicksal der Gebirgsgletscher im Anthropozän zu erörtern und über notwendige Maßnahmen $\mathrm{zu}$ beraten, die einer Stabilisierung des die Gletscher beeinflussenden Klimawandels dienen können. Die übereinstimmende Feststellung dieser Gruppe stellt eine Warnung an die Menschheit dar, sie ist ein Aufruf zu schnellem Handeln - um die globale und die regionale Erwärmung abzuschwächen, um die Gebirgsgletscher und andere verletzliche Ökosysteme zu schützen, um nationale und lokale Klimarisiken zu erkennen und um sich auf die Anpassung an solche Klimaeinwirkungen vorzubereiten, die nicht abgeschwächt werden können. Die Gruppe stellt ebenfalls fest, dass eine weitere bedeutende menschengemachte Gefahr für das Klimasystem in der Bedrohung durch Nuklearkriege besteht, die durch den schnellen und weitreichenden Abbau der globalen nuklearen Waffenbestände verringert werden kann. 


\section{Die Erde erwärmt sich und die Belastungen durch den Klimawandel nehmen zu}

Es ist eindeutig, dass sich die Erde erwärmt. Der größte Teil des beobachteten Anstiegs der global gemittelten Temperatur seit der Mitte des zwanzigsten Jahrhunderts ist mit sehr hoher, d.h. mit mehr als 90\%iger Wahrscheinlichkeit das Ergebnis des beobachteten Anstiegs der anthropogenen Treibhausgaskonzentrationen. Diese Erwärmung erfolgt, obwohl Aerosole, von denen viele ebenfalls bei der Erzeugung von $\mathrm{CO}_{2}$ emittiert werden, diesen Effekt durch eine Abkühlung verschleiern.

Manche der aktuellen und der erwarteten Auswirkungen des Klimawandels beinhalten (1) den Verlust von Korallenriffen, Wäldern, Feuchtgebieten und anderen Ökosystemen, (2) eine wesentlich schnellere Ausrottung von Arten als im historischen Durchschnitt und (3) einen Mangel an Wasser und Nahrung für viele gefährdete Völker. Der Anstieg des Meeresspiegels und die Zunahme von stärkeren Sturmwellen bedrohen ungeschützte Ökosysteme und Völker, vor allem solche, die auf tiefgelegenen Inseln und als Küstennationen leben. Der hier diskutierte Verlust der Gebirgsgletscher bedroht die Bevölkerung an den Unterläufen der Flüsse, vor allem während der Trockenzeit, in der Gletscherabflüsse am dringendsten benötigt werden.

\section{Die Gletscher der Erde gehen zurück: Gründe und Folgen}

Der auf breiter Front erfolgende Verlust von Eis und Schnee in den Gebirgsgletschern der Welt ist einer der deutlichsten Beweise für den globalen Wandel im Klimasystem. Das gegenwärtige Schwinden der Gebirgsgletscher verursacht mehr als $1 \mathrm{~mm}$ Meeresspiegelanstieg pro Jahr, das entspricht etwa einem Drittel der beobachteten Rate. Im unmittelbar zurückliegenden Zeitabschnitt des Anthropozäns resultiert der größere Teil der Gletschermassen- und Gletscherlängenverluste in den tropischen, gemäßigten und polaren Regionen aus dem beobachteten Anstieg der Treibhausgase und der Zunahme von Partikeln, die das Sonnenlicht absorbieren, wie z.B. Ruß aus nicht vollständiger Verbrennung, und Staub, der durch den Landoberflächenwandel verursacht wird.

Wie in dem Bericht des „Intergovernmental Panel on Climate Change“ (IPCC) vom Jahr 2007 dargestellt wird, zeigen Extrapolationen aus Massenbilanzstudien an 400 ausgewählten Gletschern in der ganzen Welt eine derzeitige mittlere Dickenabnahme von etwa $0.7 \mathrm{~m}$ Wasseräquivalent. Verglichen mit dem Stand in der Mitte der 1970-er Jahre hat sich die Gleichgewichtslinie zwischen Akkumulations- und Ablationsgebiet eines Gletschers in den meisten Gebirgszügen um einige hundert Meter nach oben verlagert. Bei vielen Gletschern in tiefer gelegenen Gebirgen liegt die Schneegrenze am Ende des Sommers oberhalb der größten Höhe der Berge, was für die Gletscher bei anhaltendem Klimawandel eine tödliche Gefahr darstellt. In vielen Regionen teilen sich die Gletscher, und das bringt die daraus entstehenden kleineren Gletscher noch näher an ihr endgültiges Ende.

Gletscherflächen werden auf der ganzen Welt kleiner, die höchsten Verlustraten treten in tieferen Lagen auf. Große Gletscher verlieren ihre Zungen und hinterlassen instabile Moränen und aufgestaute Seen mit bruchgefährdeten Ufern wie den Imja See in Nepal. Solche anfälligen Barrieren waren bereits in der Vergangenheit gebrochen und hatten 
Überschwemmungen verursacht, welche die schon vorher gefährdete Infrastruktur der armen Gemeinden am Unterlauf verheerten.

Im Westen von Nordamerika vergrößert der menschliche Eingriff die Staubfracht aus den Wüsten des Coloradoplateaus und des Great Basin, was den Schnee verdunkelt und damit die Schneesaison in den Rocky Mountains von Colorado um vier bis sieben Wochen verkürzt. Die Staubteilchen verstärken auch die Erwärmung der Atmosphäre, indem sie Sonnenstrahlung absorbieren. Andernorts könnten die weitverbreiteten „Braunen Wolken“ aus der nicht vollständigen Kohleverbrennung einen großen Einfluss ausüben, so z.B. im Himalaya. Es existieren nur wenige - und in manchen Fällen gar keine - Energie- und Massenbilanzstudien, welche die Wirkungen dieses Industrierußeffekts auf Schnee und Eis in so abgelegenen Gebirgsregionen quantifizieren. Die Auswirkungen, die wir mittlerweile dank genauer Messungen im Westen von Nordamerika verstehen, liefern einen Einblick in die Reaktion von Schnee und Gletschereis in anderen, ähnlich betroffenen Gebieten.

Menge und Geschwindigkeit des Gletschermassenverlustes sind in verschiedenen Gebieten unterschiedlich, und das gleiche gilt für die damit verbundenen Auswirkungen auf die jahreszeitliche Wasserverfügbarkeit in Nachbartälern und benachbarten Tiefländern. In Gebieten mit warmen und trockenen Jahreszeiten wie Zentralasien stellen die Berge und ihre Gletscher und Winterschneerücklagen „Wasserschlösser“ dar. Sie speichern Wasser für Millionen Menschen, was aber zu Trugschlüssen führen kann. Gletschermassenverluste können vorübergehend $\mathrm{zu}$ einem Ansteigen des Abflusses von langfristig gespeichertem Wasser führen; dieser Vorgang wurde in mehreren Einzugsgebieten beobachtet. Mit der weiteren Abnahme der ursprünglichen Gletscher wird aber auch der Abfluss unweigerlich abnehmen.

Gebirgsgletscher haben eine weitere wichtige Funktion: sie speichern detaillierte Informationen über vergangene Klimate und über die Möglichkeiten der Gletscher, auf verschiedene Klimavariablen zu reagieren. Damit sind Gletscher wichtige Werkzeuge im Verständnis der früheren und jetzigen Klimadynamik. Das ganze Potential von Gebirgsgletschern als Instrument der Klimaforschung wird erst allmählich verstanden. Zusätzlich erforderliche Forschung, die Unsicherheiten reduziert, dominierende Prozesse umreißt und regionale Auswirkungen quantifiziert, könnte sich in erheblichem Maße bezahlt machen. Es ist an der Zeit, den Gebirgsgletschern mehr sorgfältige Beachtung zu schenken, bevor ihre Archive für immer verloren sind.

\section{Die Vermeidung von ,gefährlichen anthropogenen Eingriffen“ erfordert klare und verpflichtende Klimaziele}

Ziel einer Klimapolitik ist es, die Treibhausgasemissionen auf einem Niveau zu stabilisieren, das eine ,gefährliche anthropogene Störung des Klimasystems“ verhindert und „es den Ökosystemen erlaubt, sich auf natürliche Weise an den Klimawandel anzupassen, eine Bedrohung der Nahrungsmittelerzeugung $\mathrm{zu}$ verhindern und eine nachhaltige wirtschaftliche Entwicklung zu ermöglichen“, wie es im Artikel 2 der UNKlimarahmenkonvention festgelegt wurde. 
Der Temperatur-Grenzwert zur Vermeidung „gefährlicher menschlicher Eingriffe“ wird derzeit bei einer Erwärmung um $2^{\circ} \mathrm{C}$ im Vergleich zum vorindustriellen Niveau vorgeschlagen, obwohl viele Wissenschaftler die Auffassung vertreten und viele Nationen dem zustimmen, dass $1,5{ }^{\circ} \mathrm{C}$ eine sicherere Obergrenze darstellen. Wissenschaftliche, politische und ökonomische Überlegungen gingen in die Festlegung dieses Grenzwerts ein, der von den internationalen Klimaverhandlungen übernommen wurde. Seit 1900 A.D. hat sich die Erde bereits um $0,75^{\circ} \mathrm{C}$ erwärmt, sie könnte die $2^{\circ} \mathrm{C}$-Schwelle im Jahr 2100 A.D. sogar dann erreichen, wenn die heutigen Treibhausgaskonzentrationen nicht weiter erhöht werden und die Luftverschmutzung aus humanitären Gründen gedrosselt wird. Es besteht jedoch die Gefahr, dass die Erwärmung sogar $3^{\circ} \mathrm{C}$ übersteigen kann, wenn die Treibhausgasemissionen weiterhin im derzeitigen Maß steigen. Damit ist das Überschreiten des Klimaziels von $2{ }^{\circ} \mathrm{C}$ eine reale und ernste Möglichkeit.

\section{Zur Begrenzung der Erwärmung und der damit verbundenen Auswirkungen sind drastische Verminderungen nötig}

Die Einsicht in die Gründe des Klimawandels und seiner derzeitigen wie seiner zu erwartenden Auswirkungen ermöglicht es der Gesellschaft, durch Minderungsmaßnahmen unüberschaubare Auswirkungen $\mathrm{zu}$ vermeiden und sich an unvermeidbare Auswirkungen anzupassen. Jetzt ist es an der Zeit zu handeln, damit die Gesellschaft eine vernünftige Chance hat, unter dem Grenzwert von $2^{\circ} \mathrm{C}$ zu bleiben.

Mögliche Entschärfung durch Reduzierung der Kohlendioxidemissionen und Erweiterung der Kohlenstoffsenken: $\mathrm{CO}_{2}$ liefert den größten Einzelbeitrag zum Treibhauseffekt. Während mehr als die Hälfte des $\mathrm{CO}_{2}$ von den Weltmeeren und terrestrischen Senken innerhalb eines Jahrhunderts aufgenommen wird, verbleiben etwa 20\% in der Atmosphäre und verursachen damit eine jahrtausendlange Erwärmung. Deshalb muss jede erdenkliche Anstrengung unternommen werden, um direkte $\mathrm{CO}_{2}$-Emissionen aus fossiler Verbrennung zu verringern, ebenso wie indirekte Emissionen durch das Abholzen von Wäldern zu reduzieren. Stattdessen müssen Wälder und andere $\mathrm{CO}_{2}$-Senken so schnell wie möglich ausgebaut werden, um die $\mathrm{CO}_{2}$-bedingten extrem langfristigen Erwärmungsprozesse und die damit verbundenen Auswirkungen zu verhindern.

Mögliche Entschärfung durch die Verringerung kurzlebiger, nicht $\mathrm{CO}_{2}$-bedingter Klimaantriebe: Der zweite Teil einer integrierten Entschärfungsstrategie besteht darin, diejenigen Klimaantriebe zu verringern, die nur kurze Verweildauern in der Atmosphäre aufweisen. Diese umfassen schwarzen Kohlenstoffruß, troposphärisches Ozon und seine Vorläufersubstanz Methan, und Fluorkohlenwasserstoff (H-FKW). Industrieruß und troposphärisches Ozon üben eine starke Wirkung sowohl auf die regionale als auch die globale Erwärmung aus. Eine Reduzierung dieser kurzlebigen Klimaantriebe durch den Einsatz von bereits vorliegenden Technologien kann die globale Erwärmungsrate in der zweiten Hälfte dieses Jahrhunderts signifikant senken, und unter der Voraussetzung, dass $\mathrm{CO}_{2}$ ebenfalls verringert wird, würde die Erwärmungsrate in der Arktis um 2/3 zurückgehen.

Eine lokale Verringerung der Luftverschmutzung kann jedes Jahr etwa 2 Millionen Leben retten, die Getreideproduktivität erhöhen und die Aufnahmefähigkeit von Pflanzen für Kohlenstoff wiederherstellen. Ein intelligenter Umgang mit Industrieruß sollte Teil einer integrierten Aerosolstrategie sein, um sicher zu stellen, dass die dadurch bedingte Erwärmung schneller abnimmt als der Kühlungseffekt durch andere Aerosole. In vielen Gebieten gibt es ein echtes Potential zur Verringerung von Industrieruß und Staubbelastungen, die beide die 
Gletscher- und Schneeschmelze beschleunigen; dies könnte geschehen durch (1) die Verringerung der Ruß-Emissionen aus traditionellen Kochstellen, indem man sie durch energieeffizientere und schadstoffärmere Herde ersetzt; (2) durch das Ausfiltern von Ruß bei der Dieselverbrennung und (3) durch eine erneute Befestigung von Wüstenflächen und anderen Böden, um Staubemissionen zu reduzieren.

Fluorkohlenwasserstoffe sind synthetische Gase und gehören in vielen Ländern zu den am schnellsten wachsenden Klimaantrieben. Auf Grund des Montrealer Protokolls über Stoffe, welche die Ozonschicht schädigen, kann die Produktion und der Einsatz von Fluorkohlenwasserstoffen stufenweise abgebaut werden, während die nachfolgenden Emissionen vom Kyoto Protokoll abgedeckt werden. Dies würde zu einer Reduzierung des $\mathrm{CO}_{2}$-Äquivalents um 100 Gigatonnen $\mathrm{CO}_{2}$ im Jahr 2050 oder früher führen. Das Montrealer Protokoll wird weithin als der beste Umweltvertrag der Welt angesehen; mit ihm wurde die Produktion von 98\% von annähernd 100 chemischen Verbindungen eingestellt, die den Fluorkohlenwasserstoffen ähneln, was einer klimatisch wirksamen Gesamtreduzierung um 135 Gigatonnen zwischen 1990 und 2010 entspricht.

Zusammenfassend wird festgehalten, dass Luftverschmutzung und Klimawandel immer noch als zwei verschiedene Probleme betrachtet werden, obwohl es sich tatsächlich um die gleiche Geißel handelt. Luftverschmutzung und Treibhausgase weisen die gleichen Emissionsquellen auf, und eine kombinierte Vorgehensweise verringert die Kosten für Gegenmaßnahmen gegen diese beiden Bedrohungen der menschlichen Gesundheit und des gesellschaftlichen Wohlergehens. Entsprechend den Forderungen der Wissenschaft müssen diese Minderungsstrategien an allen Fronten so intensiv wie möglich betrieben werden. Gemeinsam bieten sie die Möglichkeit, das Klimasystem auf einen sicheren Zustand zurückzuführen und klimabedingte Ungerechtigkeiten zu verringern. Aber die Zeit ist kurz. Schon bald könnten die Erwärmung und ihre Folgen auf das Erdsystem unumkehrbar werden, die verursacht werden durch die über Jahrtausende in der Atmosphäre verbleibenden kumulierten $\mathrm{CO}_{2}$-Emissionen.

\section{Die Anpassung muss jetzt beginnen}

Aufgrund des Zeitversatzes zwischen Abschwächungsmaßnahmen und Klimareaktion muss selbst bei einer letztendlich erfolgreichen Verringerung mit starken klimatischen Einwirkungen und möglicherweise inakzeptablen Risiken auf verletzliche Ökosysteme und die Bevölkerungen gerechnet werden. Deshalb muss bereits jetzt, zusätzlich zur Reduktion, eine Anpassung beginnen und mit allen Kräften betrieben werden.

Wir können uns nicht an Änderungen anpassen, die wir nicht verstehen können, deshalb beginnt jegliche Anpassung mit einer Bewertung. Eine Voraussetzung zur Verstärkung regionaler und lokaler Kompetenzen beim Verständnis der Auswirkungen des Klimawandels auf Natur und Gesellschaft besteht in einer internationalen Initiative für die Beobachtung und Modellierung von Gebirgssystemen und ihren Wassereinzugsgebieten mit hoher räumlicher Auflösung, naturgetreuer Topographie und für das Hochgebirge adäquaten Prozessen. 


\section{Gletscheruntersuchungen müssen ausgeweitet und verbessert werden}

Wir müssen die bisher noch nicht vollständig geklärten, maßgeblichen Klima- und Strahlungsantriebe bei Gebirgsgletschern mit deren entsprechenden Rückmeldungen charakterisieren. Unter anderem müssen wir unser Wissen über die regionalen Unterschiede von Gletscherreaktionen auf der ganzen Erde in Hinsicht auf regionale Änderungen des Klimas und absorbierender Luftverunreinigungen erweitern. Unsere Beobachtungen der Volumina der Gletscher, des Niederschlags und der daraus folgenden Veränderungen in alpinen Einzugsgebieten sind höchst begrenzt, was wiederum unsere Möglichkeiten zur Schaffung von zukünftigen Abfluss-Szenarien einschränkt. Unsere Klimamodelle können das stark gegliederte Gelände in Gebirgsregionen nicht räumlich auflösen, deshalb werden Niederschlag, Temperaturänderungen und Aerosolfracht nur schlecht in diesen Modellen wiedergegeben. Die Modellierung und langfristige Registrierung der Zusammenhänge zwischen den Änderungen in vergletscherten Kopfeinzugsgebieten und der Wasserverfügbarkeit im Unterlauf steht ebenfalls erst am Anfang.

Einer der Gründe für die - abgesehen von Gletscherlänge und -größe - nur spärlich vorhandenen Messreihen in den Hochgebirgen des Himalaya oder der Anden liegt in der Abgelegenheit und gefährlichen Natur einer Arbeit oberhalb von $6000 \mathrm{~m}$ Meereshöhe. Derzeitige Fernerkundungstechniken können Änderungen in der Ausdehnung von Gletschern und Schnee aufspüren, aber sie liefern keine quantitativen Angaben zu den jeweiligen Antriebsmechanismen, ebenso wenig wie sie wichtige Schnee- und Eiseigenschaften wie z.B. Korngröße, örtliche Verunreinigungen und Flüssigwassergehalt an der Oberfläche bereit stellen. Jedoch werden es luft- und satellitengestützte abbildende Spektrometer bald ermöglichen, räumlich zusammenhängende Messungen dieser Oberflächeneigenschaften zu machen. In Verbindung mit Beobachtungen bei ausgedehnteren Feldmesskampagnen und lokalen Energie- und Massenbilanzmessungen werden abbildende Spektrometer in Zukunft verwendet, um die nächste Generation von hoch aufgelösten Gletschermassenbilanzmodellen zu erstellen und zu validieren. Quantitative Beobachtungen sind hierfür der Schlüssel.

\section{Geoengineering: Weitere Forschungen und internationale Einschätzungen sind notwendig}

Geoengineering stellt keinen Ersatz für eine Abschwächung des Klimawandels dar. Viele Fragen müssen noch beantwortet werden, die z.B. potentielle Irreversibilitäten und Ungleichheiten in den regionalen Auswirkungen betreffen, bevor man Geoengineering mit gutem Gewissen in Erwägung ziehen kann. Es gibt keine dezidierte internationale Bewertungsmethode zur Frage des Geoengineering. Dafür benötigt man einen Konsens aller an der Maßnahme beteiligten, der auf breiter Ebene erfolgen muss und auf höchsten Standards wie z.B. dem IPCC-Modell aufbaut. Die Grundlage für eine solche Bewertung muss eine wesentlich breitere wissenschaftliche Untersuchung sein als sie bisher hat durchgeführt werden können.

Es mag klug erscheinen, Geoengineering in Betracht $\mathrm{zu}$ ziehen, falls unumkehrbare und katastrophale Klimafolgen nicht durch Verminderung und Anpassung gehandhabt werden können. Bevor irgendeine Maßnahme durchgeführt werden kann, muss ein System von 
Steuerungsmaßnahmen entwickelt werden, das die Risiken und die Vorteile von Geoengineering abwägt; zur Abschätzung der tragbaren Risiken muss ein transparenter, auf einer breiten Übereinstimmung basierender Entscheidungs-Findungs-Prozess entwickelt werden.

\section{Privatbürger und Nationen stehen in der Pflicht, jetzt zu handeln}

Die Menschheit hat das Zeitalter des Anthropozäns geschaffen und muss jetzt damit leben. Dazu bedarf es eines neuen Bewusstseins für die Gefahren, die menschliche Handlungen für die Erde und ihre Systeme beinhalten, und hier insbesondere für die Gebirgsgletscher. Das erlegt uns eine neue Pflicht zur Verminderung dieser Gefahren auf. Versagen wir bei der Abschwächung des Klimawandels, so verletzen wir unsere Pflicht gegenüber den Schwachen dieser Erde. Dies umfasst sowohl die von der Wasserversorgung durch Gebirgsgletscher abhängigen als auch die vom steigenden Meeresspiegel und stärkeren Sturmwellen bedrohten Menschen. Unsere Aufgabe beinhaltet die Pflicht, verletzlichen Gesellschaften bei den Anpassungen an unvermeidliche Änderungen zu helfen. Alle Nationen müssen sicherstellen, dass ihre Maßnahmen durchschlagend sind und unverzüglich erfolgen, um dem wachsenden Einfluss und der steigenden Gefahr des Klimawandels zu begegnen und um katastrophale unumkehrbare Folgen zu vermeiden.

Wir rufen alle Menschen und Nationen auf, die schwerwiegenden und möglicherweise unumkehrbaren Auswirkungen der globalen Erwärmung $\mathrm{zu}$ erkennen, die durch die anthropogene Emission von Treibhausgasen und anderen Umweltschadstoffen ebenso wie durch Veränderungen von Wäldern, Feuchtgebieten, Grünland und anderen Landnutzungsarten verursacht werden. Wir appellieren an alle Nationen, unverzüglich wirksame und ausreichende Maßnahmen zu entwickeln und umzusetzen, um die Ursachen und Auswirkungen des Klimawandels auf Gemeinschaften und Ökosysteme unter Einbeziehung der Gebirgsgletscher und ihrer Einzugsgebiete zu verringern, da wir uns dessen bewusst sind, dass es nur eine gemeinsame Heimat für uns alle gibt. Wenn wir jetzt im Sinn unserer gemeinschaftlichen, wiewohl unterschiedlich verteilten Verantwortung handeln, bejahen wir unsere Verpflichtung gegenüber unseren Mitmenschen und übernehmen die Verantwortung für einen Planeten, der mit dem Geschenk des Lebens gesegnet ist. Wir sind verpflichtet, die Versorgung aller Bewohner dieses Planeten mit täglichem Brot, frischer Atemluft und sauberem Trinkwasser sicherzustellen. Denn wenn wir Gerechtigkeit und Frieden wollen, müssen wir unseren Lebensraum schützen.

Ajai, L. Bengtsson, D. Breashears, P.J. Crutzen, S. Fuzzi, W. Haeberli, W.W. Immerzeel, G. Kaser, C. Kennel, A. Kulkarni, J. Mittelstraß, R. Pachauri, T.H. Painter, J. Rabassa, V. Ramanathan, A. Robock, C. Rubbia, L. Russell, M. Sánchez Sorondo, H.J. Schellnhuber, S. Sorooshian, T.F. Stocker, L.G. Thompson, O.B. Toon, D. Zaelke 